\title{
Adjective ordering in Tagalog: A cross-linguistic comparison of subjectivity-based preferences
}

\author{
Suttera Samonte \& Gregory Scontras*
}

\begin{abstract}
Previous studies have shown that speakers have robust adjective ordering preferences. For example, in English, big red apple is strongly preferred to red big apple. Recently, Scontras et al. (2017) showed that an adjective's distance from the noun it modifies is best predicted by the adjective's subjectivity, with less subjective adjectives preferred closer to the modified noun. However, this finding was limited to English. The current study investigates the status of subjectivity-based adjective ordering preference in Tagalog, a language that forms its modification structures with the conjunction-like LINKER particle. Using Tagalog translations of the original English materials, we show that subjectivity predicts ordering preferences in Tagalog, as it does in English.
\end{abstract}

Keywords. Tagalog; adjective ordering; LINKER; subjectivity

1. Introduction. Adjective ordering preferences determine the relative order of multi-adjective strings. When speakers form multi-adjective strings (e.g., big red apple), these preferences ensure that the ordering of the adjectives is non-arbitrary. Curiously, the very same preferences documented in English have been reported in a host of different languages, including Hungarian, Telugu, Mandarin Chinese, and Dutch (e.g., Martin 1969; Hetzron 1978; Dixon 1982; Sproat \& Shih 1991). Recently, Scontras et al. (2017) showed that adjective subjectivity is a robust predictor of ordering preferences in English: less subjective adjectives occur closer to the modified noun. The current study investigates the extent to which this pattern holds in a language with a diverging modification strategy. To that end, we examine ordering preferences in Tagalog.

Unlike English, Tagalog adjectives require a linking particle (-ng/na) in order to participate in modification structures (Foley 1975; Rubin 1994; Kaufman 2009). Some authors analyze the semantic contribution of LINKER similarly to that of conjunction (Rubin 1994, Scontras \& Nicolae 2014). However, in English, conjunction has been claimed to neutralize adjective ordering preferences (Ford \& Olson 1975; Byrne 1979). We therefore set out to determine (i) whether Tagalog possesses ordering preferences in the presence of LINKER, and, if so, (ii) to what extent adjective subjectivity predicts those preferences. The paper is structured as follows. In Section 2, we survey the empirical findings regarding subjectivity-based ordering preferences in English, then provide relevant background on modification structures in Tagalog. We then present the findings from two experiments. First, in Section 3, we document adjective ordering preferences in Tagalog. Next, in Section 4, we measure Tagalog adjective subjectivity. In Section 5, we compare the results of the two experiments in order to determine whether

\footnotetext{
*We gratefully acknowledge the support of the Linguistic Society of America's Committee on Ethnic Diversity in Linguistics, who awarded a travel grant to Suttera Samonte so that she could present this work at the annual meeting in New York. Authors: Suttera Samonte, University of California, Irvine (suttera.samonte@gmail.com), Gregory Scontras, University of California, Irvine (g.scontras@uci.edu).
} 
subjectivity predicts ordering preferences in Tagalog. Section 6 concludes with a comparison of our Tagalog results and the English results from Scontras et al. (2017).

2. Background. In this section, we briefly survey the empirical and theoretical terrain of adjective ordering preferences. We then turn to Tagalog, showing how modification structures ostensibly deviate from those in English.

2.1 Subjectivity-BASED ORDERING PREFEREnCES. Adjective ordering preferences have been the subject of targeted inquiry for more than half a century now, and with good reason: why should we continue to find the same preferences everywhere we look? It comes as no surprise, then, that the literature is flush with answers to this question.

Grammatical approaches attempt to specify the knowledge underlying the observed preferences. These approaches assume the existence of discrete lexical-semantic classes of adjectives (e.g., size, color, shape, etc.; Dixon 1982), which inhabit specialized syntactic projections (Cinque 1994; Scott 2002; Laenzlinger 2005). These class projections are ordered with respect to one another, which explains the robust preferences we observe cross-linguistically. However, what goes unexplained is why the various classes should be ordered in the way we find them. Moreover, as the literature on adjective ordering demonstrates, it is non-trivial to arrive at a consensus on the inventory of adjective classes, let alone the specific adjectives that inhabit them (cf., e.g., Dixon 1982; Kingsbury \& Wellman 1986; Sproat \& Shih 1991; Scott 1998).

Rather than specifying the linguistic representation of ordering preferences, psychological approaches have attempted to identify the factors that predict the specific preferences we observe; the proposed factors often relate to aspects of adjective meaning. For example, Sweet (1898) proposed that the adjectives closer to the modified noun are "closer to the noun in meaning" or have a more "specialized meaning." In the phrase big red apple, Sweet's theory would imply that the adjective red is more closely tied to the meaning of apple, whereas big does not enjoy the same tight connection-it can describe a wider range of nouns (see also Ziff 1960). Similarly, Whorf (1945) argued that adjectives placed closer to the noun "described more inherent properties" of the noun. Returning to the big red apple, Whorf's account suggests that the property of being red is more directly related to the property of being an apple than the property of being big. In other words, if something is an apple, it is likely to be red, but less likely to be big.

The trouble with many of these psychological approaches to adjective ordering lies in operationalizing the psychological factors at play, such that they can be measured and evaluated. Recently, Scontras et al. (2017) met this challenge by testing the hypothesis that adjective subjectivity is a robust predictor of ordering preferences (Quirk et al. 1985; Hetzron 1978; Tucker 1998; Hill 2012). According to the subjectivity hypothesis, less subjective adjectives are preferred closer to the modified noun. One way of evaluating adjective subjectivity is by determining the extent to which judgments of the relevant property can be seen as a matter of opinion. In the case of red, opinion is unlikely to enter into whether some object holds the appropriate color. However, with big, opinion is more likely to be a factor in judgments of relative size. Thus, in big red apple, red is less subjective than big, which is why it occurs closer to the modified noun.

Scontras et al. (2017) tested the subjectivity hypothesis in English using a series of exper- 
iments. First, they measured ordering preferences for a set of 26 adjectives by having participants choose which of two multi-adjective phrases sounded more natural (e.g., big red apple vs. red big apple). The authors validated this behavioral measure of ordering preferences by comparing it with the ordering regularities present in English corpora. The behavioral measure tracked the corpus measure $\left(r^{2}=0.83,95 \%\right.$ CI $\left.[0.63,0.90]\right)$, leading the authors to conclude that they had a good handle on the English preferences.

To determine adjective subjectivity, Scontras et al. began with a direct measure, asking participants how "subjective" a given adjective was on a scale from "completely objective" to "completely subjective." The authors validated their subjectivity measure with a so-called "faultless disagreement" task (Kölbel 2004; Barker 2013; Kennedy 2013; MacFarlane 2014). In the task, participants were presented with a scenario where two speakers disagreed on the trait of some object (e.g., whether some apple was red). Participants judged whether both speakers could be correct while disagreeing, or whether one of them had to be wrong. To the extent that two speakers can disagree about a property judgment without one of them being wrong, the property admits that degree of faultless disagreement, which serves as an index of subjectivity. Indeed, the two measures were highly correlated $\left(r^{2} 0.91,95 \%\right.$ CI $\left.[0.86,0.94]\right)$.

To determine the extent to which subjectivity predicts ordering preferences, Scontras et al. compared the preferences they measured with the subjectivity scores they gathered. Subjectivity accounted for $85 \%$ of the variance in the ordering preferences $\left(r^{2}=0.85,95 \%\right.$ CI $[0.75$, 0.90]). In other words, subjectivity does indeed predict ordering preferences. The authors explored the robustness of this finding by repeating their methodology on a larger set of 78 adjectives drawn from naturalistic multi-adjective strings in the Switchboard corpus. Again, the authors found subjectivity to be a reliable and robust predictor of ordering preferences in English: less subjective adjectives are preferred closer to the modified noun.

Recent work has followed up on the empirical findings of Scontras et al. (2017) by offering explanations for why subjectivity should play the role it does in adjective ordering (Hahn et al. 2018; Simonič 2018; Scontras et al. to appear). So far, all of the proposed accounts demonstrate how ordering adjectives with respect to decreasing subjectivity maximizes the probability of communicative success. Simonič (2018) and Scontras et al. (to appear) assume that semantic composition follows the hierarchical structure of nominal modification so that adjectives closer to the modified noun compose earlier; with this assumption, they show how composing less subjective adjectives earlier (i.e., closer to the noun) leads to a greater chance of successful classification of the intended referent. Hahn et al. (2018) assume that meaning composition proceeds with the linear uptake of words in real time; the authors also assume that speakers are more likely to forget words they have heard further in the past. With the goal of sharing speaker opinions via non-restrictive modification, the authors show how subjectivity-based orderings better preserve the intended message under pressure from fallible memory. Although they differ on the details, all three accounts predict that pressures for successful communication should apply universally, such that we would expect to find subjectivity-based ordering preferences cross-linguistically.

2.2 Adjectival modificAtion in TAgAlOG. With clear evidence concerning the role of subjectivity in English ordering preferences, our interest now shifts to the question of whether 


\begin{tabular}{ll}
\hline$\checkmark$ LINKER & $*$ LINKER \\
\hline attributive adjective & predicative adjective \\
adverbial modifier & predicative adverbial \\
nominal modifier & predicative nominal \\
relative clause & matrix clause \\
\hline
\end{tabular}

Table 1: Distribution of LINKER from Scontras \& Nicolae (2014)

subjectivity plays a similar role in languages that use ostensibly different means of adjectival modification. Specifically, we investigate the status of ordering preferences in Tagalog. Tagalog (Malayo-Polynesian: Western Malayo-Polynesian) is the national language of the Philippines, with approximately 30 million speakers, not including those who live abroad or who learned Tagalog as a second language. Tagalog resembles English in that modifying adjectives occur pre-nominally (although not always; cf. Schachter \& Otanes 1972; Rubin 1994; Shih \& Zuraw 2017). Similarly, in multi-adjective strings, adjectives occur before the modified noun. For example, in (1), the adjectives malaki 'big' and pula 'red' occur before mansanas 'apple':

(1) malaki-ng pula-ng mansanas

big-LK red-LK apple

'big red apple'

The key difference between Tagalog and English is the use of the so-called LINKER. In (1), the linking particle -ng obligatorily intervenes between an adjective-noun combination (i.e., between pula and mansanas), as well as between the two adjectives (i.e., between malaki and pula). In fact, in all cases of adjectival modification, LINKER appears; however, with predicative adjectives, LINKER is obligatorily absent. Compare (2a) and (2b):
a. pula-ng mansanas
red-LK apple
'red apple'
b. $\quad$ pula $(*-n g)$ ang mansanas
red-LK TOP apple
'The apple is red.'

The form of LINKER depends on the phonological shape of the word it follows: words that end in vowels (or an alveolar nasal or glottal stop) take $-n g$, while words ending in consonants take $n a$, as in (3).

(3) maliit na itim na mansanas

small LK black LK apple

'small black apple'

The distribution of LINKER is not limited to adjectival modification. In their study of LINKER, Scontras \& Nicolae (2014) identify the distribution in Table 1. On the basis of this distribution, Scontras \& Nicolae conclude that LINKER surfaces in the context of non-saturating semantic composition, or modification (cf. Rubin 1994; Sabbagh 2009). In other words, when- 
ever two elements of the same semantic type compose (e.g., an adjective and a noun, both type $\langle e, t\rangle$ ), LINKER intervenes. Following Rubin (1994), Scontras \& Nicolae (2014) propose that LINKER surfaces in the context of modification because it discharges the semantics of modification; they give LINKER the denotation in (4a), and the structure in (4b). Extending this proposal to multi-adjective strings, we arrive at the structure in (5) for (1).

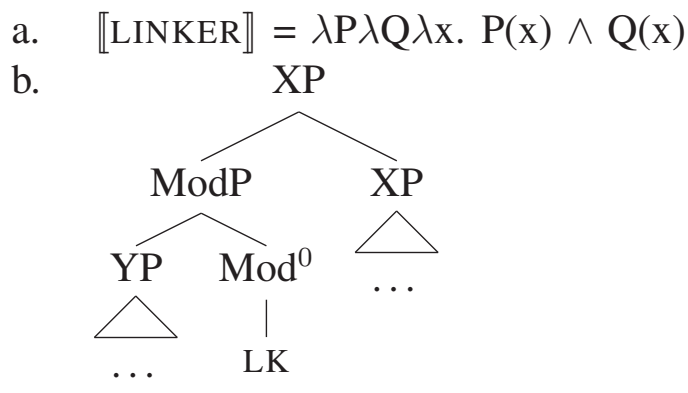

(5)

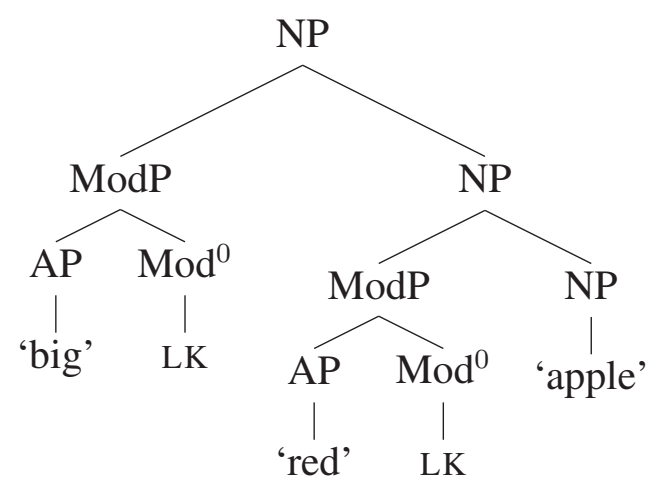

Other analyses of LINKER make do without positing functional structure or even a semantic entry for LINKER (cf. the morphological flagging account of Chung \& Ladusaw 2004). Our aim is not to decide between the proposed accounts here. Rather, we highlight the proposal in (4) because of the implications it might have for ordering preferences in Tagalog. Of particular interest is the striking similarity between LINKER and conjunction. The semantics in (4a) appears very similar, if not identical to the semantics for conjunction in the nominal domain: two properties are conjoined to yield a new property that incorporates both of the original properties. Importantly, at least in English, conjunction has been claimed to neutralize adjective ordering preferences (e.g., Ford \& Olson 1975; Byrne 1979). In other words, although speakers strongly prefer big red apple to red big apple, with conjunction, red and big apple is claimed to be acceptable (but see Rosales \& Scontras 2019 for evidence of subjectivity-based preferences even with conjunction in English).

We therefore face the following question: if Tagalog requires a conjunction-like LINKER to mediate the composition of multi-adjective strings, and if conjunction does indeed neutralize adjective ordering preferences, then does LINKER neutralize ordering preferences in Tagalog? To answer this question, we extend the methodology from Scontras et al. (2017) to measure ordering preferences and adjective subjectivity in Tagalog.

3. Experiment 1: Measuring ordering preferences. Our first task is to determine whether Tagalog has ordering preferences in the presence of LINKER. To determine the status of Taga- 


\begin{tabular}{llllll}
\hline adjective & translation & class & adjective & translation & class \\
\hline bago & new & age & maganda & nice & quality \\
bulok & rotten & age & sira & unusable & quality \\
luma & old & age & bilog & round & shape \\
sariwa & fresh & age & cuadrado & square & shape \\
asul & blue & color & parihaba & rectangular & shape \\
berde & green & color & tatsulok & triangular & shape \\
dilaw & yellow & color & mahaba & long & size \\
itim & black & color & maikli & short & size \\
pula & red & color & malaki & big & size \\
puti & white & color & maliit & small & size \\
aluminyo & aluminum & material & makinis & smooth & texture \\
bakal & metal & material & malambot & soft & texture \\
kahoy & wooden & material & matigas & hard & texture \\
\hline noun & translation & class & noun & translation & class \\
\hline kamatis & tomato & food & lampara & lamp & furniture \\
karot & carrot & food & mesa & desk & furniture \\
keso & cheese & food & silya & chair & furniture \\
mansanas & apple & food & sopa & couch & furniture \\
saging & banana & food & telebisyon & TV & furniture \\
\hline
\end{tabular}

Table 2: Tagalog adjectives and nouns used in Experiment 1 and Experiment 2. Words in italics indicate cases where direct translation from the original English materials was not possible.

log ordering preferences, we replicated Expt. 1: Ordering preferences from Scontras et al. (2017) using Tagalog translations of the original English materials.

3.1 PARTICIPAnTS. We recruited 90 participants through Amazon.com's Mechanical Turk crowdsourcing service. All participants were compensated for their participation. On the basis of their responses to a post-test questionnaire, we identified 24 Tagalog speakers (12 female, 12 male; mean age: 32); their data were included in the analyses reported below. Of these 24 participants, 22 were born in the Philippines and 2 were born in the United States. Now, five currently reside in the Philippines, 17 reside in the United States, and two reside in some other country.

3.2 Procedure. Participants indicated their preferences for pairs of multi-adjective strings formed from 26 unique adjectives from seven semantic classes, together with ten nouns. Adjectives and nouns were direct translations of the English materials from Scontras et al. (2017); when direct translation was not possible, we substituted Tagalog words from the same semantic class. The full set of materials appears in Table 2.

Participants completed a series of 26 trials. Multi-adjective strings were chosen at random with the constraint that the two adjectives be from two separate semantic classes. The pairs 


\section{Anong tunog ng "mansanas" na naglalarawan na may natural na himig?}

"ang pulang malaking mansanas"

"ang malaking pulang mansanas"

\section{Iurong ang nararapat na panukala para alamin ang sinasabing pahayag.}

$$
\text { Susunod }
$$

Figure 1: Sample trial from Experiment 1. Participants indicated the preferred relative order of two adjectives in a multi-adjective string.

of multi-adjective strings participants saw in each trial differed in the relative order of the adjectives (e.g., pulang malaking mansanas 'red big apple' vs. malaking pulang mansanas 'big red apple'). Participants adjusted a slider with endpoints labeled with the competing multiadjective strings. On the basis of these slider ratings, we arrived at a single preferred-distance measure for each adjective; values ranged from 0 (always preferred closest to the noun) to 1 (always preferred farthest from the noun). Figure 1 presents an example trial from the experiment.

3.3 RESULTS. We computed a mean preferred-distance measure for each of the 26 adjectives by averaging ratings across participants. Figure 2 plots these preferred-distance measures grouped by adjective class. All but the age adjectives deviate significantly from the random baseline (i.e., from 0.5), suggesting that Tagalog does indeed have stable preferences. Texture, size, and quality adjectives are preferred farther from the noun on average, and color, shape, and material adjectives are preferred closer. Thus, we find strong evidence in support of stable ordering preferences in Tagalog, despite the obligatory LINKER. We will use the preferreddistance measures obtained for individual adjectives below in our analysis of adjective subjectivity. First, we need to obtain subjectivity scores for the 26 adjectives.

4. Experiment 2: Measuring adjective subjectivity. Having observed stable ordering preferences in Tagalog, our next task is to measure adjective subjectivity. To do so, we replicated Expt. 1: Faultless disagreement validation from Scontras et al. (2017) using the Tagalog materials from Experiment 1.

4.1 PARTICIPANTS. We recruited 45 participants who did not take part in Experiment 1 through Amazon.com's Mechanical Turk. All participants were compensated for their participation.

On the basis of their responses to a post-test questionnaire, we identified 11 Tagalog speakers ( 8 female, 3 male; mean age: 29); their data were included in the analyses below. Of the 11 Tagalog-speaking participants, ten were born in the Philippines and one was born in the United States. Six currently reside in the Philippines, four in the United States, and one in some other country. 


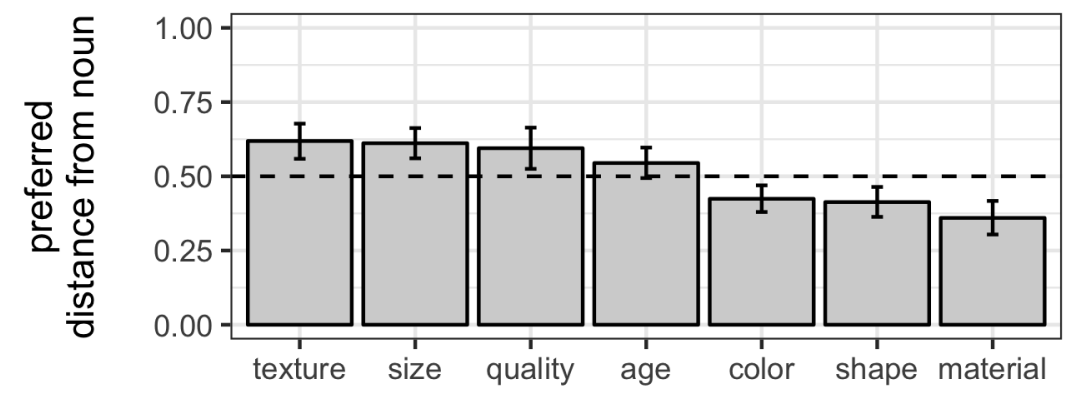

adjective class

Figure 2: Naturalness ratings from Experiment 1 grouped by adjective semantic class. Higher values indicate that a class's adjectives are preferred farther from the modified noun; lower values indicate that a class's adjectives are preferred closer. The dashed line indicates chance level, or the absence of stable preferences. Error bars represent bootstrapped $95 \%$ confidence intervals drawn from 10,000 samples of the data.

4.2 Procedure. Participants encountered a series of dialogues in which two speakers disagreed about a property ascription for some object. For example, the two speakers might observe an apple. One speaker would assert that the apple is red, and the other would counter by saying that the apple is not red. Participants judged whether both speakers could be right while disagreeing, or whether one speaker must be wrong. Judgments were provided by adjusting a slider with endpoints labeled 'only one can be right' (coded as 0) and 'yes, it depends on what you believe' (coded as 1). Figure 3 presents an example trial from the experiment.

Participants completed a series of 26 trials in random order, one for each of the adjectives in Table 2; on each trial, the noun was chosen at random from the list in Table 2.

4.3 RESULTS. We computed a mean faultless disagreement score for each adjective by averaging scores across participants. Figure 4 plots these faultless disagreement scores grouped by adjective class. We will use the faultless disagreement scores for the individual adjectives in the next section, where we test whether subjectivity predicts ordering preferences in Tagalog.

5. Comparing ordering preferences with subjectivity. With estimates of the Tagalog ordering preferences and adjective subjectivity, our final task is to compare the two measures to determine the extent to which subjectivity predicts Tagalog ordering preferences. Figure 5 plots the preferred-distance measures for each of the 26 adjectives against their faultless disagreement (i.e., subjectivity) scores. Adjective subjectivity accounts for $54 \%$ of the variance in the ordering-preference data $\left(r^{2}=0.54,95 \% \mathrm{CI}=[0.22,0.74]\right)$. In other words, subjectivity does indeed predict adjective ordering preferences in Tagalog.

6. General discussion. We have found strong evidence in support of subjectivity-based adjective ordering preferences in Tagalog. Despite the obligatory presence of LINKER in Tagalog modification structures, the results of Experiment 1 demonstrate that speakers do have stable ordering preferences; these preferences determine the relative order of adjectives in multiadjective strings. After measuring adjective subjectivity with the faultless disagreement task 


\section{Progreso:}

Isaalang-alang ang sitwasyon:

Stacy at Andrew ay nakakita ng parehong mansanas.

Sabi ni Stacy: "Ang mansanas ay pula."

Sumagot si Andrew: "Mali ka. Ang mansanas ay hindi pula."

Maaaring maging pareho sila ni Stacy at si Andrew ay tama?

Isa sa kanila dapat ay tama.

Oo, depende sa anong iyong paniniwala.

Susunod

Figure 3: Sample trial from Experiment 2. Participants rated the possibility for two speakers to faultlessly disagree about a property ascription.

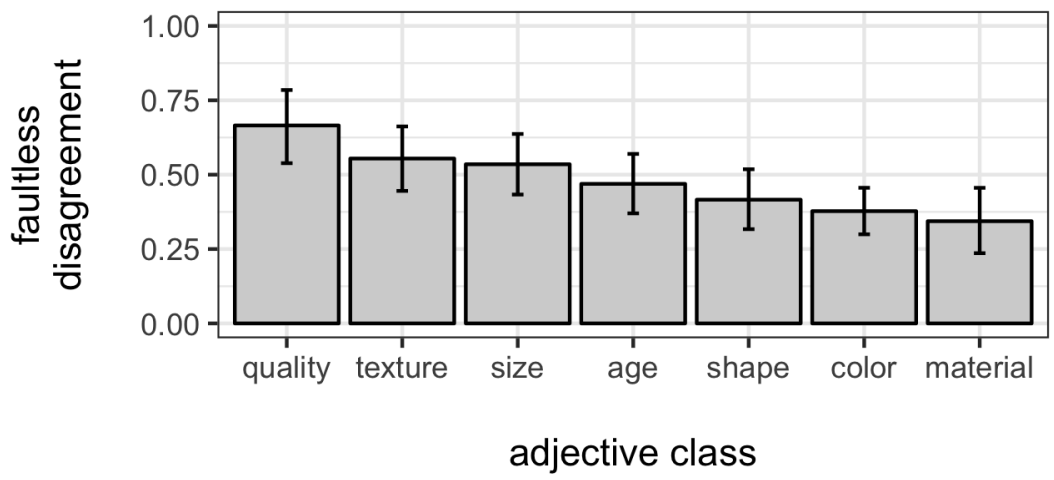

Figure 4: Faultless disagreement scores from Experiment 2 grouped by adjective semantic class. Higher values indicate that a class's adjectives are perceived as more subjective. Error bars represent bootstrapped $95 \%$ confidence intervals drawn from 10,000 samples of the data.

in Experiment 2, we found that subjectivity predicts the Tagalog preferences, as it does in English.

Although both Tagalog and English evidence subjectivity-based ordering preferences, there is a salient difference between the results from the two languages: Scontras et al. (2017) found subjectivity to be a much stronger predictor of the English ordering preferences. Using English translations of the materials tested in the current study, the authors observed that subjectivity accounted for between $85 \%$ and $88 \%$ of the variance in the ordering preferences they measured. By contrast, we have found that subjectivity accounts for $54 \%$ of the Tagalog variance-a marked departure from the English baseline.

One might wonder whether the difference we observe between English and Tagalog could be due to imprecise (or impossible) translations. There were eight adjectives in our study that were not translation equivalents with the original English materials (cf. Table 2). Perhaps these 


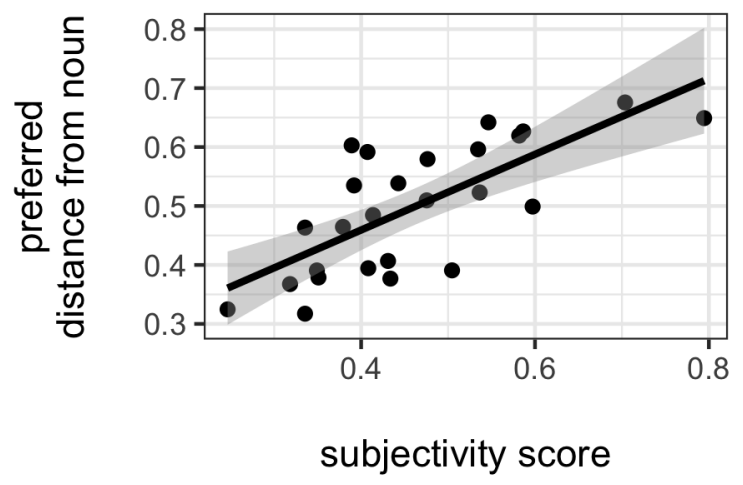

Figure 5: Ordering preferences (obtained in Experiment 1) plotted against subjectivity scores (obtained in Experiment 2) for each of the 26 adjectives tested.

eight adjectives - nearly a third of the adjectives we tested-led to the poorer performance of subjectivity relative to the English baseline. While we cannot rule out this possibility, we do consider it unlikely that the specific adjectives tested were the sole source of the lower correlation we observe in Tagalog. Rather, there is likely an additional source: the quantity and quality of our Tagalog-speaking participants.

When using Mechanical Turk to collect behavioral data, native speakers of English are ubiquitous among the participant pool; native speakers of Tagalog are not. This fact is reflected in the numbers of participants run in the current study vs. those run in the studies by Scontras et al. (2017). We used data from 24 Tagalog speakers to determine ordering preferences, and 11 Tagalog speakers to measure adjective subjectivity; Scontras et al. had double the number of English speakers for their results. This asymmetry in the number of participants could have introduced more variance in the Tagalog results, such that subjectivity was less capable of explaining it.

Another worry that separates our participants from those in the English study concerns their status as monolingual native speakers. Most speakers of Tagalog are bilingual, and in many cases that bilingualism is imbalanced in favor of a language other than Tagalog. For speakers residing in the Philippines, it is common for Tagalog to be a second language, after one of the many Filipino dialects. And given that the majority of our participants now reside in the United States, we face the possibility that some are English-dominant heritage speakers of Tagalog (for reviews of heritage speakers and the properties of heritage langauges, see Benmamoun et al. 2013; Scontras et al. 2015; Montrul 2016; Polinsky 2018). Both situations are likely to lead to increased noise in the Tagalog intuitions we measured-noise that would decrease the strength of the correlation between ordering preferences and subjectivity.

Even with these considerations, we still find subjectivity to be a robust predictor of adjective ordering preferences in Tagalog, lending support to the universal nature of the proposed explanations discussed in Section 2: pressures toward successful communication should apply universally, such that we find subjectivity-based ordering preferences. Both the incrementalprocessing story of Hahn et al. (2018) and the hierarchical-composition story of Simonič (2018) and Scontras et al. (to appear) predict that ordering adjectives in Tagalog according to decreas- 
ing subjectivity would maximize communicative success, even in the presence of LINKER.

\section{References}

Barker, Chris. 2013. Negotiating Taste. Inquiry 56(2-3). 240-257. https://doi.org/10.1080/0020174X.2013.784482.

Benmamoun, Elabbas, Silvina Montrul \& Maria Polinsky. 2013. Heritage languages and their speakers: Opportunities and challenges for linguistics. Theoretical Linguistics 39(3-4). 129-181. https://doi.org/10.1515/tl-2013-0009.

Byrne, Brian. 1979. Rules of prenominal adjective order and the interpretation of "incompatible" adjective pairs. Journal of Verbal Learning and Verbal Behavior 18(1). 73-78. https://doi.org/10.1016/S0022-5371(79)90574-7.

Chung, Sandra \& William Ladusaw. 2004. Restriction and saturation. Cambridge, MA: MIT Press.

Cinque, Guglielmo. 1994. On the evidence for partial N-movement in the Romance DP. In R. S. Kayne, G. Cinque, J. Koster, J.-Y. Pollock, L. Rizzi \& R. Zanuttini (eds.), Paths towards universal grammar. studies in honor of Richard S. Kayne, 85-110. Washington DC: Georgetown University Press.

Dixon, R. M. W. 1982. Where have all the adjectives gone? And other essays in semantics and syntax. Berlin: Mouton.

Foley, William. 1975. Comparative syntax in Austronesian: University of California, Berkeley dissertation.

Ford, William \& David Olson. 1975. The elaboration of the noun phrase in children's description of objects. Journal of Experimental Child Psychology 19(3). 371-382. https://doi.org/10.1016/0022-0965(75)90068-5.

Hahn, Michael, Judith Degen, Noah D. Goodman, Dan Jurafsky \& Richard Futrell. 2018. An information-theoretic explanation of adjective ordering preferences. In Proceedings of the 40th annual conference of the Cognitive Science Society, London: Cognitive Science Society.

Hetzron, Robert. 1978. On the relative order of adjectives. In Hansjakob Seiler (ed.), Language universals, 165-184. T"ubingen: Narr.

Hill, Felix. 2012. Beauty before age? Applying subjectivity to automatic English adjective ordering. Proceedings of the NAACL HLT 2012 student research workshop. 11-16.

Kaufman, Daniel. 2009. Austronesian Nominalism and its consequences: A Tagalog case study. Theoretical Linguistics 35. 1-49. https://doi.org/10.1515/THLI.2009.001.

Kennedy, Christopher. 2013. Two sources of subjectivity: Qualitative assessment and dimensional uncertainty. Inquiry 56(2-3). 258-277. https://doi.org/10.1080/0020174X.2013.784483.

Kingsbury, Roy \& Guy Wellman. 1986. Longman advanced English. Harlow, Essex: Longman. K"olbel, Max. 2004. Faultless disagreement. Proceedings of the Aristotelian Society 104. 53-73. https://doi.org/10.1111/j.0066-7373.2004.00081.x.

Laenzlinger, Christopher. 2005. French adjective ordering: perspectives on DP-internal movement types. Lingua 115. 645-689. https://doi.org/10.1016/j.lingua.2003.11.003. 
MacFarlane, John. 2014. Assessment Sensitivity. Oxford: Clarendon Press.

Martin, J. E. 1969. Some competence-process relationships in noun phrases with prenominal and postnominal adjectives. Journal of Verbal Learning and Verbal Behavior 8. 471-480. https://doi.org/10.1016/S0022-5371(69)80091-5.

Montrul, Silvina. 2016. The acquisition of heritage languages. Cambridge: Cambridge University Press.

Polinsky, Maria. 2018. Heritage languages and their speakers. Cambridge: Cambridge University Press.

Quirk, Randolph, Sidney Greenbaum, Geoffrey Leech \& Jan Svartvik. 1985. A Comprehensive Grammar of the English Language. London: Longman.

Rosales, Cesar Manuel, Jr. \& Gregory Scontras. 2019. On the role of conjunction in adjective ordering preferences. In Proceedings of the Linguistic Society of America 4. 32:1-12.

Rubin, Edward J. 1994. Modification: A syntactic analysis and its consequences. Ithaca, NY: Cornell University dissertation.

Sabbagh, Joseph. 2009. Existential sentences in Tagalog. Natural Language \& Linguistic Theory 27. 675-719. https://doi.org/10.1007/s11049-009-9083-3.

Schachter, Paul \& Fe T. Otanes. 1972. Tagalog reference grammar. Berkeley: University of California Press.

Scontras, Gregory, Judith Degen \& Noah D. Goodman. 2017. Subjectivity predicts adjective ordering preferences. Open Mind: Discoveries in Cognitive Science 1(1). 53-65. https://doi.org/10.1162/OPMI a $\_0005$.

Scontras, Gregory, Judith Degen \& Noah D. Goodman. to appear. On the grammatical source of adjective ordering preferences. Semantics and Pragmatics .

Scontras, Gregory, Zuzanna Fuchs \& Maria Polinsky. 2015. Heritage language and linguistic theory. Frontiers in Psychology 6(1545). https://doi.org/10.3389/fpsyg.2015.01545.

Scontras, Gregory \& Andreea Cristina Nicolae. 2014. Saturating syntax: Linkers and modification in Tagalog. Lingua 149. 17-33. https://doi.org/10.1016/j.lingua.2014.05.005.

Scott, Gary-John. 1998. Stacked adjectival modification and the structure of nominal phrases. SOAS Working Papers in Linguistics and Phonetics 8. 59-89.

Scott, Gary-John. 2002. Stacked adjectival modification and the structure of nominal phrases. In Guglielmo Cinque (ed.), The cartography of syntactic structures, volume 1: Functional structure in the DP and IP, 91-120. Oxford: Oxford University Press.

Shih, Stephanie S. \& Kie Zuraw. 2017. Phonological conditions on variable adjective and noun word order in Tagalog. Language 93. e317-e352. https://doi.org/10.1353/lan.2017.0075.

Simonič, Mihael. 2018. Functional explanation of adjective ordering preferences using probabilistic programming. University of T"ubingen MA thesis.

Sproat, Richard \& Chilin Shih. 1991. The cross-linguistic distribution of adjective ordering restrictions. In C. Georgopoulos \& R. Ishihara (eds.), Interdisciplinary approaches to language: Essays in honor of S.-Y. Kuroda, 565-593. Dordrecht: Kluwer Academic Publishers.

Sweet, Henry. 1898. A New English grammar, logical and historical. London: Clarendon Press. 
Tucker, Gordon. 1998. The lexicogrammar of adjectives: A systemic functional approach to lexis. London: Cassell Academic.

Whorf, Benjamin Lee. 1945. Grammatical categories. Language 21(1). 1-11. https://doi.org/10.2307/410199.

Ziff, Paul. 1960. Semantic analysis. Ithaca, NY: Cornell University Press. 